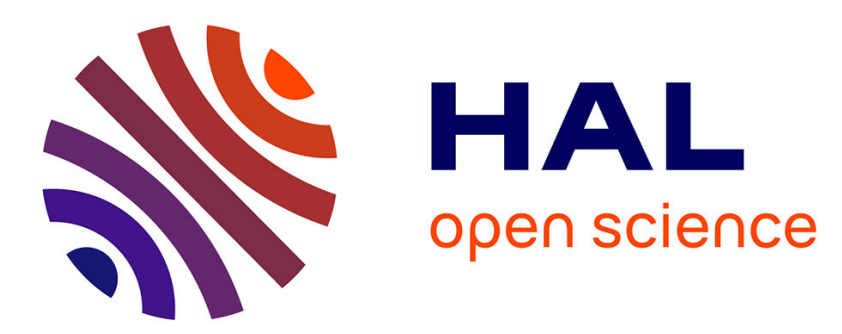

\title{
Dérive temporelle post-irradiation dans les dosimètres MOS de rayonnement
}

\author{
Gérard Sarrabayrouse, A. Bellaouar, P. Rossel
}

\section{To cite this version:}

Gérard Sarrabayrouse, A. Bellaouar, P. Rossel. Dérive temporelle post-irradiation dans les dosimètres MOS de rayonnement. Revue de Physique Appliquée, 1986, 21 (2), pp.131-137. 10.1051/rphysap:01986002102013100 . jpa-00245418

\section{HAL Id: jpa-00245418 https://hal.science/jpa-00245418}

Submitted on 1 Jan 1986

HAL is a multi-disciplinary open access archive for the deposit and dissemination of scientific research documents, whether they are published or not. The documents may come from teaching and research institutions in France or abroad, or from public or private research centers.
L'archive ouverte pluridisciplinaire HAL, est destinée au dépôt et à la diffusion de documents scientifiques de niveau recherche, publiés ou non, émanant des établissements d'enseignement et de recherche français ou étrangers, des laboratoires publics ou privés. 


\title{
Dérive temporelle post-irradiation dans les dosimètres MOS de rayonnement
}

\author{
G. Sarrabayrouse, A. Bellaouar et P. Rossel \\ Laboratoire d'Automatique et d'Analyse des Systèmes du C.N.R.S., \\ 7, avenue du Colonel Roche, 31077 Toulouse Cedex, France
}

(Reçu le 10 octobre 1985, accepté le 12 novembre 1985)

\begin{abstract}
Résumé. - Les propriétés de la dérive post-irradiation dans les transistors MOS utilisés comme dosimètres sont étudiées. Il est montré que cette dérive, probablement due à une injection tunnel des électrons du canal, est une fonction fortement décroissante de l'épaisseur de la grille. Une évaluation des erreurs commises lors de la mesure de faibles débits de dose permet de définir les limites d'utilisation de ce type de dosimètre.
\end{abstract}

\begin{abstract}
The properties of the post-irradiation annealing in MOS transistors used as dosimeter are studied. It is shown that the observed shift, probably caused by tunnel injection of electrons from the transistor channel, is a decreasing function of the insulator thickness. The limits of such a dosimeter when a very low dose rate is measured are evaluated.
\end{abstract}

\section{Introduction.}

L'utilisation de transistors MOS comme dosimètre des rayonnements ionisants a été proposée en 1970 par W. J. Pock et al. [1]. Depuis cette date, plusieurs études ont été consacrées à ce détecteur; le support expérimental était constitué par des composants élaborés suivant des technologies standard et comportant une couche isolante d'épaisseur inférieure à $1500 \AA[2,3]$. Récemment, nous avons rapporté des résultats expérimentaux concernant un dosimètre MOS ayant une grande épaisseur d'oxyde $D_{\text {ox }}\left(1000 \AA<D_{\text {ox }}<7000 \AA\right)$ et élaboré suivant un processus technologique assurant un bon compromis entre sensibilité $\left(>1 \mathrm{~V} / \mathrm{Gray}\left(\mathrm{SiO}_{2}\right)\right.$ ) et stabilité [4]. Une sensibilité élevée permet une mesure de faibles doses obtenues sous un faible débit; toutefois, le phénomène de dérive temporelle du composant durant une irradiation à faible débit ou après irradiation lors de mesures intégrées prend alors une importance particulière.

Cette dérive peut être due à deux phénomènes :

- Un transport des trous créés par le rayonnement dans la couche isolante, vers l'interface $\mathrm{SiO}_{2}-\mathrm{Si}[5,6]$. Cette dérive est dite rapide et intervient durant des temps de l'ordre de quelques secondes après irradiation.

- Une compensation des charges volumiques créées par une injection tunnel d'électron du semiconducteur vers l'isolant [7]. Cette dérive, dite lente, intervient durant des temps plus longs et suit une loi de variation logarithmique avec le temps en l'absence de création d'états de surface et de non-uniformités latérales [8].

L'objectif de cet article est d'étudier les propriétés des dérives temporelles dans les transistors à forte épaisseur d'oxyde ayant été irradiés et d'évaluer les erreurs qui peuvent être commises de ce fait lors de mesure de doses. Nous nous limiterons au cas des dérives lentes qui seules présentent de l'importance ici.

\section{Etude expérimentale des dérives.}

Les composants étudiés sont des transistors MOS canal $\mathrm{N}$ dont le processus technologique a été décrit en détail $[4,9]$. Les couches d'oxyde sont obtenues par oxydation thermique du silicium à $1150^{\circ} \mathrm{C}$ sous oxygène sec et recuites à la même température durant $15 \mathrm{~min}$ sous argon. Les épaisseurs obtenues se situent dans la gamme $1000 \AA-6800 \AA$. La grille métallique est en chrome déposé par évaporation thermique.

La caractérisation de la dérive post-irradiation est effectuée en mesurant l'évolution temporelle de la tension de seuil du transistor par une méthode déjà décrite [10]. L'irradiation des composants est effectuée à l'aide d'un générateur de rayons X Aequivolt 300/150 délivrant un débit de dose contrôlable entre $2,5 \times 10^{-2}$ Gray $\left(\mathrm{SiO}_{2}\right) \min ^{-1}$ et 3,7 Gray $\left(\mathrm{SiO}_{2}\right)$ $\min ^{-1}$.

Durant l'irradiation, lorsqu'un champ électrique $E_{\mathrm{ir}}$ est appliqué dans l'oxyde, la tension de seuil $V_{\mathrm{T}_{0}}$ des transistors évolue linéairement avec la dose de rayon- 
nement comme le montre la figure 1 . Après arrêt de l'irradiation, la tension de seuil dérive en sens inverse en suivant une loi qui tend vers une loi logarithmique du

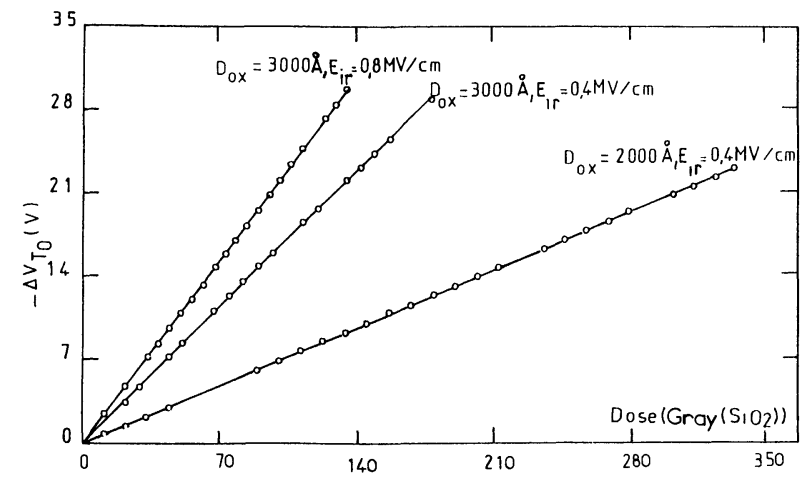

Fig. 1. - Variation typique $\Delta V_{\mathrm{T}_{0}}$ de la tension de seuil d'un transistor MOS sous rayonnement en fonction de la dose, de l'épaisseur de la couche de silice $D_{\mathrm{ox}}$ et du champ électrique $E_{\text {ir. }}$.

[Typical variation $\Delta V_{\mathrm{T}_{0}}$ of the threshold voltage as a function of the irradiation dose, the silica layer thickness $D_{\mathrm{ox}}$ and the electric field $E_{\mathrm{ir}}$ ]

temps comme le montre typiquement la figure 2. Sur cette figure $\Delta V_{\mathrm{T}}^{\mathrm{R}}$ est la différence entre la variation totale $\Delta V_{\mathrm{T}}$ de la tension de seuil par rapport à sa valeur pré-irradiation et la variation $\Delta V_{\mathrm{T}_{0}}$ durant l'irradiation. Par ailleurs, la dérive $\Delta V_{\mathrm{T}}^{\mathrm{R}}(t)$ est sensiblement proportionnelle à la dose de rayonnement reçue durant l'irradiation. En effet, la figure 3 représente le rapport $\Delta V_{\mathrm{T}}^{\mathrm{R}}(t) / \Delta V_{\mathrm{T}_{0}}$ en fonction du temps et de la dose de rayonnement reçue. Il apparaît sur cette figure que ce rapport est faiblement affecté par la dose.

Deux paramètres sont particulièrement importants en ce qui concerne le dosimètre MOS; ce sont l'épais-

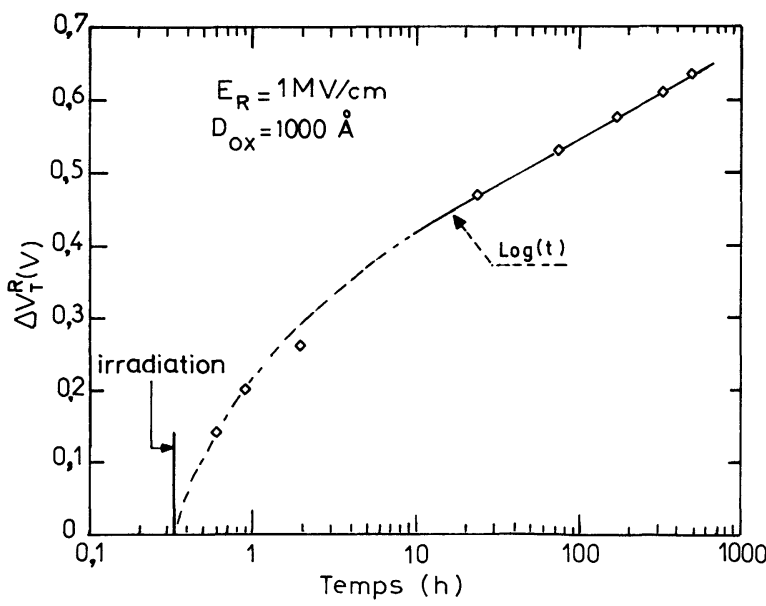

Fig. 2. - Variation typique de la tension de seuil après irradiation.

[Typical variation of the threshold voltage after irradiation.]

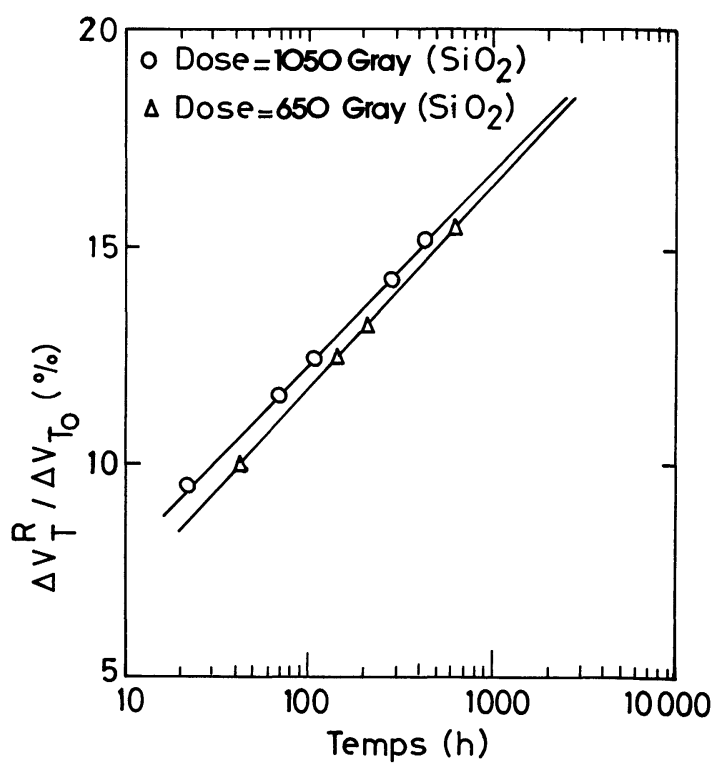

Fig. 3. - Influence de la dose sur la dérive relative postirradiation. $D_{\mathrm{ox}}=1000 \AA, E_{\mathrm{R}}=1 \mathrm{MV} \mathrm{cm}-1$.

[Influence of the irradiation dose on the post-irradiation annealing.]

seur de la couche isolante $D_{\mathrm{ox}}$ et le champ électrique appliqué. En effet, ces deux paramètres contrôlent dans de larges proportions la valeur de la sensibilité du dosimètre ou l'amplitude de la dégradation à saturation.

L'influence de l'épaisseur sur l'amplitude des dérives est représentée sur la figure 4 qui montre le poids

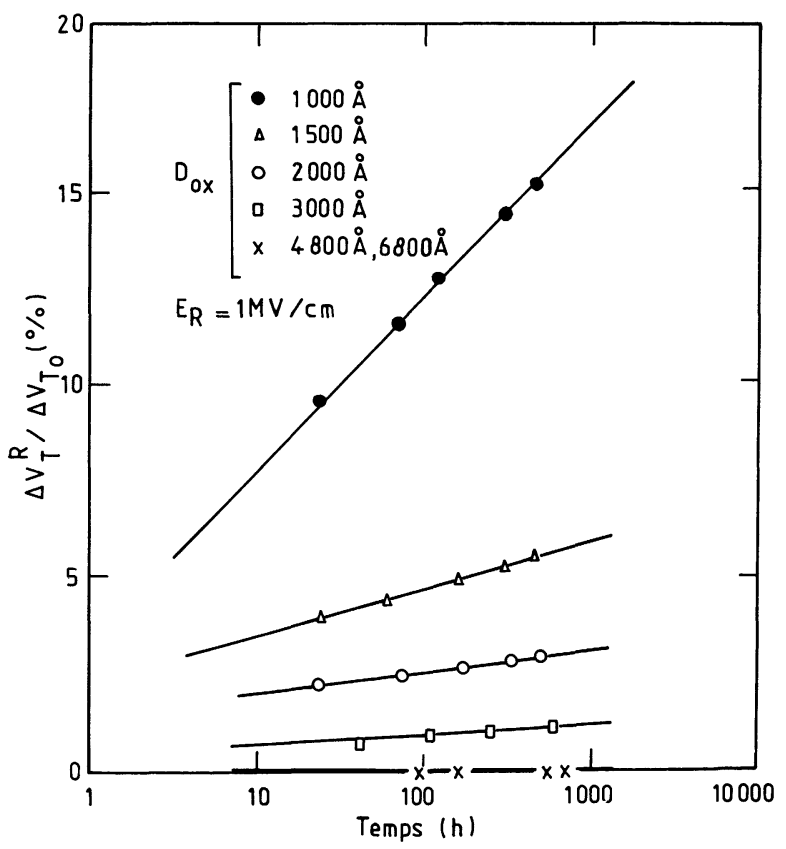

Fig. 4. - Influence de l'épaisseur de la couche isolante $D_{0 x}$ sur l'amplitude de la dérive relative post-irradiation.

[Influence of the insulator layer thickness on the post-irradiation annealing.] 
important de ce paramètre sur la dérive à long terme. Un fait particulièrement intéressant est que cette dérive décroît avec l'épaisseur alors que la sensibilité croît $[4,9,11]$. Donc une épaisseur importante de la couche isolante assure à la fois une meilleure sensibilité et une meilleure stabilité du dosimètre. En particulier les transistors comportant une couche isolante de $6800 \AA ̊$ ont une sensibilité voisine de $1,5 \mathrm{~V} / \mathrm{Gray}\left(\mathrm{SiO}_{2}\right)$ et comme le montre la figure 4 , une stabilité de la dégradation meilleure que $0,1 \%$ pour $10^{3}$ heures de dérive à la température ambiante.

Peu de résultats concernant l'étude des dérives - et particulièrement sur l'influence de l'épaisseur de l'isolant - ont été publiés $[8,12,13]$. Les résultats obtenus ici se comparent difficilement à ceux de la littérature où généralement l'influence de l'épaisseur va en sens inverse $[8,12]$. Le phénomène de dérive est intimement lié aux caractéristiques des pièges à électrons et donc du processus technologique. En particulier la gamme généralement étudiée se situe en dessous de $1000 \AA$. Dans la publication [8] on peut observer une décroissance de $\frac{\Delta V_{\mathrm{T}}^{\mathrm{R}}}{\Delta V_{\mathrm{T}_{0}}}$ avec l'épaisseur pour les deux plus fortes épaisseurs testées. Dans la référence [12] d'une part, le mécanisme de dérive mis en cause n'est pas seulement la compensation des charges créées car on observe une dérive relative supérieure à $100 \%$, mais de plus, la dérive est enregistrée à une température de $100^{\circ} \mathrm{C}$. Or, il a été suggéré que suivant la température, le mécanisme impliqué soit différent [14].

L'influence du champ électrique appliqué $E_{\mathrm{R}}$ lors de la dérive est typiquement illustrée par la figure 5 . Bien que l'ordre de grandeur de $\Delta V_{\mathrm{T}}^{\mathrm{R}}$ ne change pas fortement avec $E_{\mathrm{R}}$, l'influence de ce paramètre n'apparaît cependant pas négligeable. De ce fait, un mécanisme physique de la dérive mettant en cause la

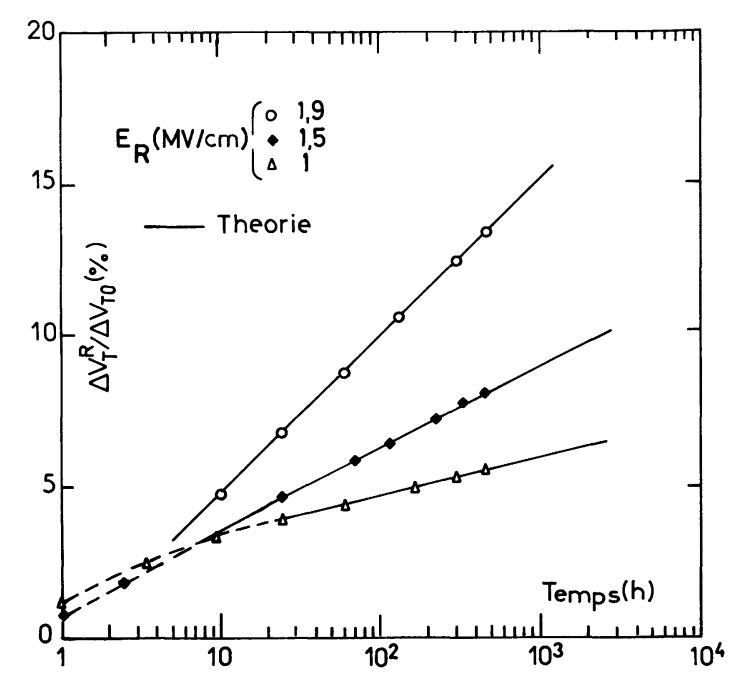

Fig. 5. - Influence du champ électrique appliqué dans l'oxyde durant la dérive. $D_{o x}=1500 \AA$.

[Influence of the electric field applied accross the insulating layer.] relaxation thermique des porteurs piégés dans l'oxyde ne peut être retenu dans le cadre des expériences effectuées ici [15].

Enfin, toujours dans l'optique d'obtenir un dosimètre stable, nous avons examiné l'influence d'un recuit post-oxydation sur l'amplitude des dérives. En effet, un recuit haute température est connu pour accroître la sensibilité au rayonnement d'un composant MOS [11]. Il est donc important de s'assurer qu'il n'entraîne pas une dégradation de la stabilité du composant. La figure 6 montre que ce recuit a une faible influence (bénéfique) sur la dérive post-irradiation.

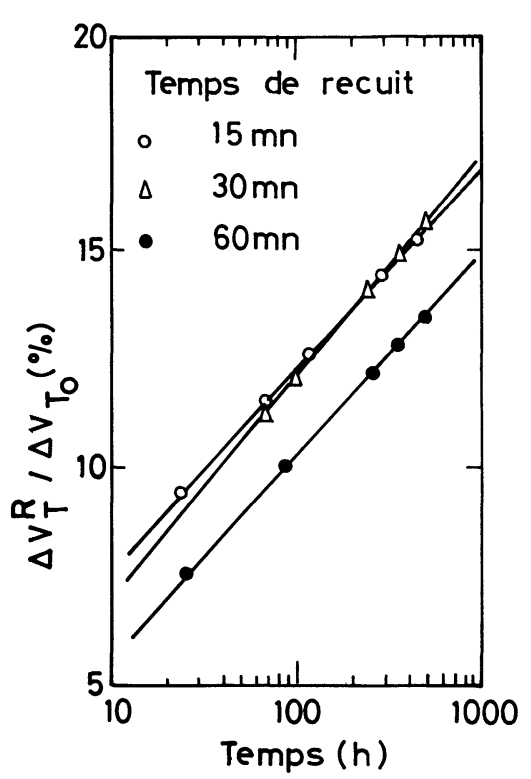

Fig. 6. - Influence du temps de recuit sur la dérive; $D_{\mathrm{ox}}=1000 \AA$. Recuit post-oxydation sous argon à $1150^{\circ} \mathrm{C}$.

[Influence of the post oxydation annealing time.]

\section{Modélisation de la dérive.}

3. 1 Modélisation PHYSIQUE. - La variation linéaire de la dégradation sous rayonnement avec la dose reçue $[4,9]$, la dépendance logarithmique du temps de la dérive post-irradiation ainsi que l'absence de guérison complète de la dégradation indiquent que ni la densité d'états de surface, ni les non-uniformités latérales de potentiel (LNU) ne contrôlent les dérives post-irradiation [8]. Dans ces conditions, un mécanisme basé sur la neutralisation des charges créées par le rayonnement près de l'interface $\mathrm{SiO}_{2}-\mathrm{Si}$ avec des électrons injectés depuis le semiconducteur par effet tunnel direct a été proposé par F. B. Mc Lean et al. [7]. Ce mécanisme a par ailleurs été invoqué avec succès pour rendre compte des dérives temporelles de structures métal-oxyde-antimoniure d'indium dont l'isolant comportait des centres pièges natifs [16].

Considérons un transistor MOS à canal $\mathrm{N}$ dont l'oxyde contient une densité volumique $N_{\mathrm{T}}$ de pièges à électrons créés par l'irradiation [17] et répartis de 
manière uniforme énergétiquement et spatialement dans une zone située à la distance $x_{01}$ de l'interface $\mathrm{SiO}_{2}-\mathrm{Si}$ (voir Fig. 17).

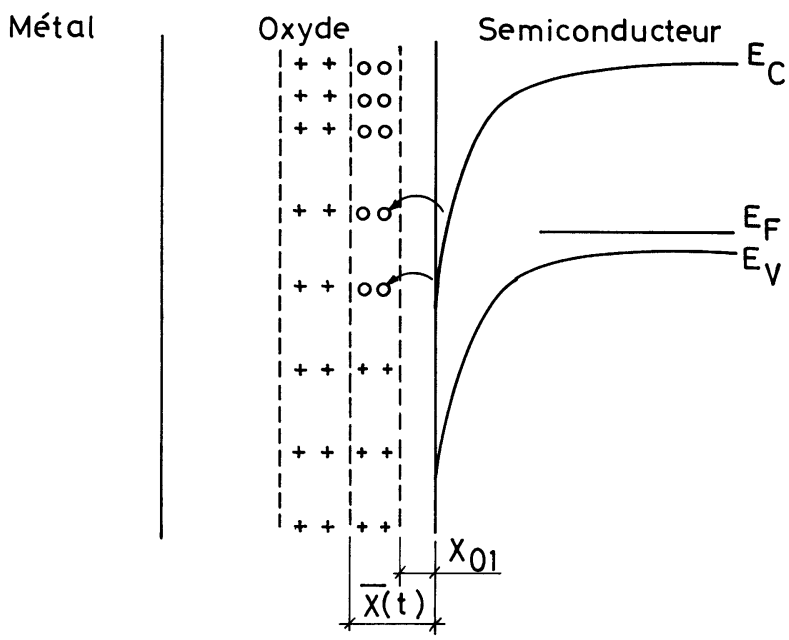

Fig. 7. - Représentation schématique du mécanisme de dérive.

[Schematics of the annealing mechanism.]

Lors d'une polarisation positive de l'électrode de grille, une densité élevée d'électrons libres est présente à l'interface $\mathrm{SiO}_{2}-\mathrm{Si}$. Ces électrons peuvent transiter par effet tunnel avec une probabilité temporelle $P$ vers l'isolant et être captés par les centres pièges compensant ainsi la charge positive présente.

L'équation d'évolution du taux d'occupation en électron $f_{\mathrm{t}}$ de ces centres s'écrit :

$$
\frac{\partial f_{\mathrm{t}}}{\partial t}(E, x, t)=P\left[f_{\mathrm{s}}(E, t)-f_{\mathrm{t}}(E, x, t)\right]
$$

où $E$ est l'énergie du piège, $x$ sa position dans l'isolant par rapport à l'interface $\mathrm{SiO}_{2}-\mathrm{Si}, f_{\mathrm{s}}$ le taux d'occupation à l'énergie $E$ à l'interface $\mathrm{SiO}_{2}$-Si dans le semiconducteur.

Deux approximations peuvent être faites : la première tient compte de la décroissance exponentielle de la probabilité $P$ avec la distance $x$. De ce fait, on peut considérer que le piégeage s'effectue à partir de $x_{01}$ suivant un front de charge situé en $\bar{x}(t)$ progressant au cours du temps vers le volume de l'isolant, les pièges situés entre $x_{01}$ et $\bar{x}(t)$ étant en équilibre avec le semiconducteur.

La seconde tient compte de la faible valeur de la dérive enregistrée dans nos composants $\left(\Delta V_{T_{R}} /\right.$ $\Delta V_{\mathrm{T}_{0}}<20 \%$ ). On peut considérer alors que la variation de charges au cours du temps affecte peu la densité électronique à l'interface $\mathrm{SiO}_{2}-\mathrm{Si}$ dans le semiconducteur, densité fixée uniquement par la polarisation de grille et la charge positive initiale. Dans ces conditions, on montre [9] que si la probabilité $P$ s'exprime par [18]

$$
P(x) \approx P_{0} \exp (-\lambda x)
$$

où $P_{0}$ est une constante de l'ordre de $10^{13} \mathrm{~s}^{-1}$, la position du front de charge $\bar{x}(t)$ s'exprime par :

$$
\bar{x}(t)=x_{01}+\lambda \ln \left[1+P\left(x_{01}\right) \cdot t\right]
$$

et la dérive relative de la tension de seuil par :

$$
\begin{aligned}
& \frac{\Delta V_{\mathrm{T}_{\mathrm{R}}}(t)}{\Delta V_{\mathrm{T}_{\mathrm{o}}}}=\frac{k T}{\lambda} \frac{N_{\mathrm{T}}}{N_{\mathrm{irr}}} \ln [1\left.+P\left(x_{01}\right) t\right] \times \\
& \times \ln \left[1+\frac{E_{\mathrm{F}}-E_{\mathrm{cs}}}{k_{\mathrm{T}}}\right]
\end{aligned}
$$

où $E_{\mathrm{cs}}$ obtenu par résolution du théorème de Gauss à l'interface $\mathrm{SiO}_{2}$-Si est l'énergie du bas de la bande de conduction du semiconducteur à l'interface $\mathrm{SiO}_{2}-\mathrm{Si}$, $E_{\mathrm{F}}$ l'énergie du niveau de Fermi à cette interface et $N_{\text {irr }}$ la densité par unité de surface de charges positives créées par l'irradiation déduite de la valeur de $\Delta V_{\mathrm{T}_{0}}$. Un lissage des résultats expérimentaux de la figure 5 au moyen de la relation (4) a été obtenu pour des valeurs des paramètres $x_{01}$ et $N_{\mathrm{T}}$ représentées dans le tableau I. Le paramètre $N_{\text {irr }}$ a été déterminé à l'aide de la valeur de $\Delta V_{\mathrm{T}_{0}}$.

Tableau I.

\begin{tabular}{cccc}
$V_{\mathrm{G}}(\mathrm{V})$ & $-\Delta V_{\mathrm{T}_{0}}(\mathrm{~V})$ & $X_{01}(\AA)$ & $N_{\mathrm{T}}\left(\mathrm{cm}^{-3} \mathrm{eV}^{-1}\right)$ \\
\hline 15 & 20 & 35,1 & $4 \times 10^{19}$ \\
\hline 22 & 25,7 & 38 & $8,3 \times 10^{19}$ \\
\hline 28 & 27 & 38,6 & $1,4 \times 10^{19}$
\end{tabular}

Il apparaît que la dépendance logarithmique avec le temps de la dérive, sa dépendance vis-à-vis de la tension ainsi que son ordre de grandeur peuvent être rendus par le modèle simple pour des valeurs des paramètres raisonnables. En effet, la valeur de $x_{01}$ est compatible avec les « distances tunnel » inférieures typiquement à 50-60 $\AA$ dans la silice. La densité volumique de pièges n'a pas fait l'objet d'études antérieures, certainement du fait des difficultés expérimentales lors de la détermination de la distribution spatiale des charges créées par le rayonnement. La densité utilisée ici pour accorder les résultats expérimentaux et théoriques $\left(5 \times 10^{19}-10^{20} \mathrm{~cm}^{-3}\right)$ peut apparaître comme étant élevée. Cependant D. J. Di Maria et al. [19] ont montré que le centroïde de la charge positive créée par irradiation aux rayons $\mathrm{X}$ est responsable $\mathrm{du}$ piégeage des électrons [20] et se situe aux alentours de $X_{\mathrm{c}}=50 \AA$. Dans ces conditions, si l'on considère une charge répartie uniformément entre $\left(X_{c}-X_{01}\right)$ et $\left(X_{\mathrm{c}}+X_{01}\right)$ avec une densité volumique $N_{\mathrm{T}}$, la densité superficielle effective de piège à électron se situe dans la même décade que $N_{\text {irr }}$. Compte tenu de l'incertitude sur le facteur $\lambda$ qui a été pris égal à sa valeur théorique $10^{8} \mathrm{~cm}^{-1}$ [21], l'ordre de grandeur de $N_{\mathrm{T}}$ apparaît comme étant acceptable. 
3.2 INFLUENCE DE LA DÉRIVE DURANT L'IRRADIATION. - Dans le cas d'une irradiation à très faible débit de dose - en environnement spatial où le débit peut être inférieur à $0,01 \mathrm{Gray} /$ heure par exemple - la présence d'une guérison du composant peut perturber l'indication fournie par le dosimètre dès lors que la dérive est du même ordre de grandeur que le taux de création de charges positives sous irradiation. En conséquence, il apparaît important de déterminer l'influence de cette dérive durant une irradiation à faible débit.

L'ensemble des résultats expérimentaux concernant une irradiation à débit de dose dit « fort » pendant une durée faible devant la période où la réponse du composant est observée, montre (Figs. 1 et 3) que celle-ci est linéaire avec la dose de rayonnement.

En conséquence, on peut considérer le composant comme un système linéaire dont la réponse a une impulsion de rayonnement de dose $D_{0}$ serait donnée par la loi logarithmique $\Delta V_{\mathrm{T}}^{D_{\mathrm{o}}}(t)$ enregistrée longtemps après l'irradiation telle que :

$$
\Delta V_{\mathrm{T}}^{D_{\mathrm{o}}}(t)=\Delta V_{\mathrm{T}_{0}}+\Delta V_{\mathrm{T}}^{\mathrm{R}}(t)=A \ln \left(t / t_{0}\right)-C .
$$

En conséquence, la réponse à une irradiation de durée non impulsionnelle peut être donnée par [21]

$$
\Delta V_{\mathrm{T}}(t)=\int_{0}^{t} \dot{D}(\tau) \frac{\Delta V_{\mathrm{T}}^{D_{0}}}{D_{0}}(t-\tau) \mathrm{d} \tau
$$

où $\dot{D}(\tau)$ est le débit de dose durant l'irradiation.

Dans le cas d'une irradiation à débit constant $B$ durant une durée $t_{\mathrm{ex}}$, la réponse du composant compte tenu de (5) et (6) est donnée par :

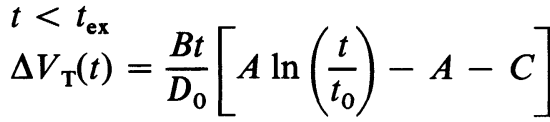

$$
\begin{aligned}
& \begin{array}{l}
t>t_{\mathrm{ex}} \\
\Delta V_{\mathrm{T}}(t)
\end{array}=\left[A \left(x^{\prime} \ln \left(\frac{x^{\prime}}{x^{\prime}-1}\right)+\ln \left(x^{\prime}-1\right)+\right.\right. \\
& \left.+\ln \left(\frac{t_{\text {ex }}}{t_{0}}\right)\right)-A-C
\end{aligned}
$$

où $x^{\prime}=t / t_{\mathrm{ex}}$ et $t_{0}$ est une constante de normalisation.

Un exemple d'accord entre la relation (8) et l'expérience quel que soit l'instant post-irradiation considéré est représenté sur la figure 8. Les constantes $A$ et $C$ dans (8) ont été obtenues compte tenu de (5) à partir de l'équation de la partie linéaire de la courbe.

Cet accord apparaît satisfaisant et valide l'approche proposée. Il n'a pas été possible de valider la relation (7). En effet, compte tenu d'une part des débits de dose à notre disposition et d'autre part de la faible amplitude des dérives $(<20 \%)$ même pour les composants à couche isolante de $1000 \AA$, nous n'avons pu mettre en évidence l'influence de la dérive durant l'irradiation.

3.3 DÉBIT DE DOSE MINIMUM MESURABLE. - Considérons un composant irradié sous un débit de dose $B$ très

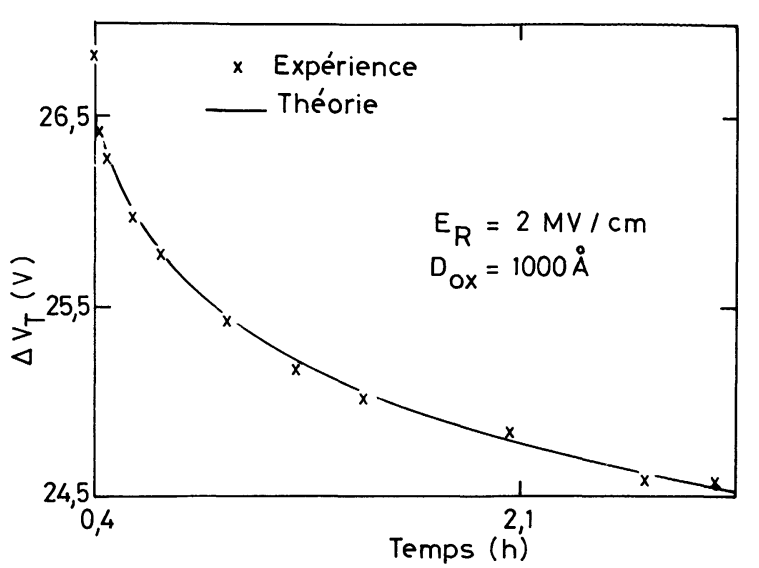

Fig. 8. - Accord théorie-expérience sur la dérive à moyen terme; temps d'irradiation : $0,4 \mathrm{~h} ; A=0,7108 \mathrm{~V}$; $C=25,25 \mathrm{~V}$.

[Comparison between theory and experiment on the medium term annealing.]

faible. A l'instant $t$ la dose intégrée reçue est $D=B t$ et produit un déplacement $\Delta V_{\mathrm{T}}(\tau)$ de la tension de seuil donné par la relation (7) ci-dessus. Lors de la mesure, ce déplacement est interprété en terme d'une dose $D_{0}$ reçue à débit élevé dans un laps de temps très court et donné par la relation (5) évaluée pour $t=t_{0}$ [22]. D'où

$$
\Delta V_{\mathrm{T}}(t)=\Delta V_{\mathrm{T}}^{D_{0}}\left(t=t_{0}\right)
$$

soit en tenant compte $\operatorname{des}(7)$ et (5)

$$
\left(\frac{B t}{D_{0}}-1\right)=\left[\frac{C}{-A\left[\ln \left(\frac{t}{t_{0}}\right)-1\right]+C}-1\right] .
$$

La quantité dans le premier membre représente l'erreur relative sur la valeur de la dose mesurée dans des conditions de faible débit.

Nous avons porté sur la figure 9 , les variations du rapport $B / D_{0}$ où $D_{0}$ est la dose utilisée pour l'obtention

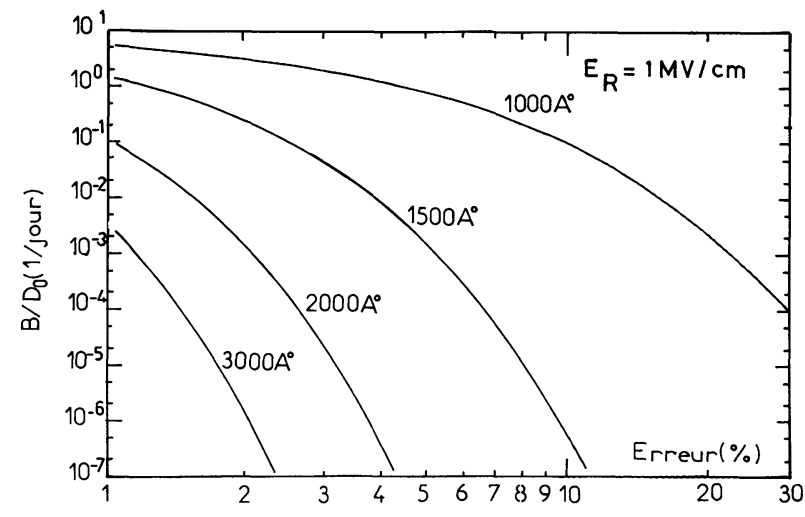

Fig. 9. - Variations du rapport entre le débit $B$ et la dose $D_{0}$ en fonction de l'erreur sur la mesure de la dose pour différentes épaisseurs $D_{\mathrm{ox}}$.

[Ratio of the dose rate to dose versus the experimental error.] 
de la caractéristique de dérive à long terme, en fonction de l'erreur sur l'évaluation de la dose. Les constantes $A$ et $C$ utilisées dans cette figure, ont été obtenues à partir des résultats expérimentaux de dérive à long terme. Ces courbes permettent par exemple, de déterminer le débit de dose minimal qu'un composant peut détecter si nous fixons l'erreur et la dose totale accumulée et inversement.

Il n'a pas été possible de vérifier expérimentalement avec précision ces résultats, du fait d'une part des caractéristiques $d u$ générateur de rayons $X$ qui n'autorisent pas des débits très faibles et d'autre part de la faiblesse des dérives.

La figure 10 représente cependant les résultats expérimentaux correspondant aux débits maximum et minimum disponibles. Dans ces conditions, les courbes de la figure 9 indiquent une erreur inférieure à $1 \%$ pour une dose arbitrairement choisie à $4,5 \mathrm{Gray}\left(\mathrm{SiO}_{2}\right)$.

Il apparaît expérimentalement sur la figure 10 une erreur inférieure à $4 \%$ qui se situe dans la « limite de la stabilité » de notre générateur fonctionnant à faible débit.

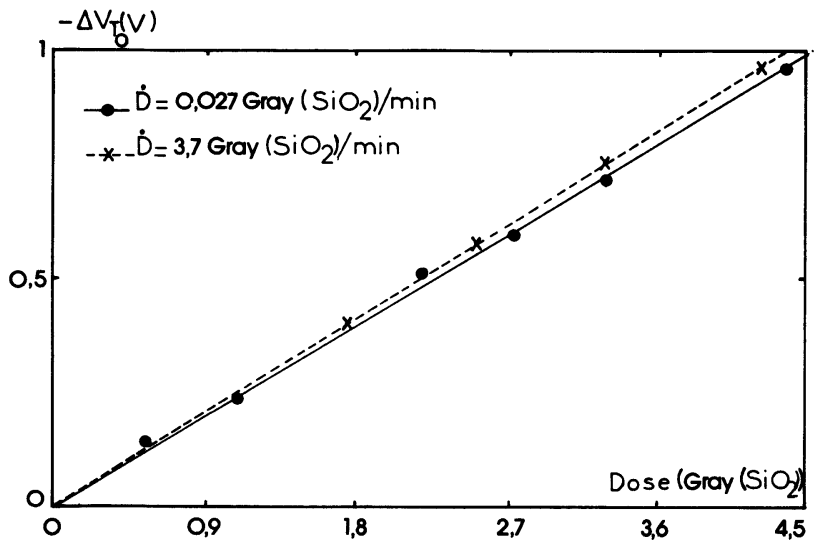

Fig. 10. - Influence du débit de dose sur la dégradation durant l'irradiation. $D_{\mathrm{ox}}=3000 \AA ; E_{\mathrm{ir}}=1 \mathrm{MV} / \mathrm{cm}$

[Influence of the dose rate on the irradiation shift.]

\section{Conclusion.}

L'étude expérimentale et théorique des dérives postirradiation a montré que celles-ci sont dues à un phénomène de piégeage d'électrons sur les charges positives ou les centres neutres dans une zone très proche de l'interface $\mathrm{SiO}_{2}-\mathrm{Si}$, les électrons transitant par effet tunnel entre la bande de conduction et l'isolant.

Dans le cadre des composants MOS étudiés, ces dérives sont d'autant plus faibles que l'épaisseur de la couche isolant est importante, ce qui correspond à une sensibilité au rayonnement croissante. Ainsi, il apparaît que les composants MOS sont bien adaptés à la dosimétrie des rayons $\gamma$ dans une large gamme de dose et de débit.

En particulier et à titre d'exemple pour une mission spatiale d'un satellite à travers la ceinture de VanAllen (altitude 500 et $600 \mathrm{~km}$ ), le débit de dose est approximativement de 0,01 Gray $\left(\mathrm{SiO}_{2}\right)$ par jour pour 'un écran en aluminium d'épaisseur égale à $2 \mathrm{~g} / \mathrm{cm}^{2}$; pour une mission de 5 ans, la dose accumulée est de $100 \mathrm{Gray}\left(\mathrm{SiO}_{2}\right)$. L'erreur sur l'évaluation de la dose liée à la dérive du composant simultanée avec la dégradation de la tension seuil, est déterminée sur la figure 11. Cette erreur est une fonction de l'épaisseur d'oxyde de grille; le cas étudié correspond à un champ de $1 \mathrm{MV} / \mathrm{cm}$ appliqué sur la grille. La courbe a été déterminée à partir de la figure 9. Cet effet de dérive est donc de moins de $5 \%$ pour des épaisseurs d'oxyde du transistor supérieures à $1500 \AA$.

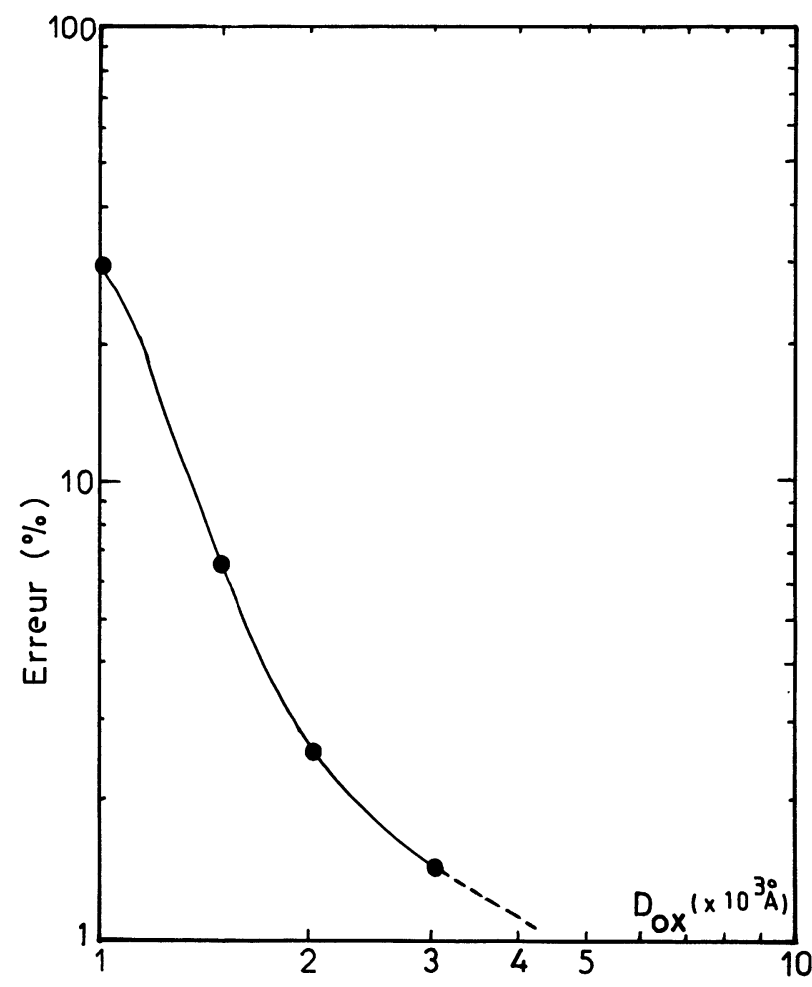

Fig. 11. - Evolution de l'erreur sur la mesure de la dose en fonction de $D_{\text {ox }}, D=0,01$ Gray $\left(\mathrm{SiO}_{2}\right) /$ jour, Dose $=$ 100 Gray $\left(\mathrm{SiO}_{2}\right)$.

[Experimental error as a function of the insulator thicknes.]

\section{Remerciements.}

Les auteurs remercient Madame F. Rossel et Monsieur Do Conto pour leur aide dans la réalisation des échantillons. 


\section{Bibliographie}

[1] Poch, W. J. and Holmes Siedle, A. G., RCA Engineer 16, no 3 (1970) 56.

[2] Dawes, W. R. and Schwauk, J. R. IEEE Trans. Nucl. Sci. N 528, 6 (1981) 4152.

[3] August, L. S., IEEE Trans. Nucl. Sci. NS 29, 6 (1982) 2000.

[4] Bellaouar, A., Sarrabayrouse, G. and Rossel, P. 13th Yougoslav. Conference on Microelectronics (MIEL 85) Ljubljana 8-10 May 1985.

[5] Mcleau, F. B., Boesch, Jr. H. E. and McGarrity, J. M., IEEE Trans. Nucl. Sci. NS 23 (1976) 1506.

[6] Srour, J. R., Othmer, S., Curtis, O. L., Jr. AND CHIU, K. Y. IEEE Trans. Nucl. Sci. NS 23 (1976) 1513.

[7] Mcleau, F. B., Boesch, Jr. H. E., Winckur, P. S., MC GaRrity, J. M. and Oswald, Jr. R. B., IEEE Trans. Nucl. Sci. NS 21 (1974) 47.

[8] Winokur, P. S. and BoesCH, Jr., H. E., IEEE Trans. Nucl. Sci. NS 28, 6 (1981) 4088.

[9] Bellaouar, A. Thèse de $3 \mathrm{e}$ cycle. Université Paul Sabatier, $\mathrm{n}^{\circ}$ 3178. Toulouse 26 juin 1985.

[10] Bellaouar, A., Sarrabayrouse, G. and Rossel, P. Influence of ionizing irradiation on the channel mobility of MOS transistors. IEE proceedings part I, Solid-State and Electron Devices, à paraître.

[11] Derbenwick, G. F. and Gregory, B. L. IEEE Trans. Nucl. Sci. NS 22, 6 (1975) 2151.

[12] Schwank, J. R. and Daves, W. R. IEEE Trans. Nucl. Sci. NS 30, 6 (1983) 4100.
[13] Derbenwick, G. F. and SAnder, H. H. IEEE Trans. Nucl. Sci. NS 24, 6 (1977) 2244.

[14] Holmes Siedle, A. and Freeman, R. Radiation effect Engineering Handbook. Final Report European Space Agency Contract $n^{\circ}$ 2871/76/NL/HP. Fulmer Research Institute (UK).

[15] Habing, D. H. and Shafer, B. D., IEEE Trans. Nucl. Sci. NS 20, 6 (1973) 307.

[16] Buxo, J., Estève, D., Farre, J., Sarrabayrouse, G. and Simonne, J., Appl. Phys. Lett. 33, no 11 (1978) 969.

[17] Aitken, J. M., Young, D. R. and Pan, K., J. Appl. Phys. 49, 6 (1978) 3386.

[18] Lundström, I. and Svensson, C., J. Appl. Phys. 43, 12 (1972) 5045.

[19] Di Maria, D. J., Weinberg, Z. A. and Aitken, J. M., J. Appl. Phys. 48, 3 (1977) 898.

[20] Aitken, J. M. and Young, D. R., J. Appl. Phys. 47, 3 (1976) 1196.

[21] PAUTER, P. F., Modulation, Noise and spectral analysis (Mc Graw-Hill) 1965, p. 77.

[22] La loi expérimentale représentée par la relation (5) est en $\ln \left(t / t_{0}\right)$ et est une limite pour $t \gg t_{0}$ de la loi théorique représentant la dérive donnée par la relation (4) qui montre une dépendance en $\ln \left(1+t / t_{0}\right)$. Dans le cadre de cette limite, le temps le plus court mesurable est $t_{0}$. Cette procédure est équivalente à celle qui consiste à considérer théoriquement une impulsion de Dirac à $t=0$. 